\title{
In ricordo di Massimo Campanini
}

\author{
Roberto Tottoli | ORCID: 0000-0002-1176-9953 \\ Università di Napoli “L'Orientale”, Dipartimento di Asia Africa Mediterraneo, \\ Napoli, Italia \\ rtottoli@unior.it
}

Con Massimo ci siamo conosciuti veramente solo quando venne a Napoli per il concorso da Ricercatore (2005) e negli anni passati all'Orientale (2005-2011). Occasione per frequentarci nei primi mesi furono le volte che rimase a dormire da me e mia moglie Francesca in una casa in cui ci si riuniva settimanalmente per lunghe cene con i colleghi. Ci si trovava con il compianto Giovanni D'Erme, ma anche con Claudio Lo Jacono, Michele Bernardini, Alberto Ventura, Giovanni Canova, Natalia Tornesello e tanti altri che si alternavano con la scusa di un corso di rilegatura che finiva però in lunghe chiacchierate.

Massimo era contento di essersi finalmente unito a noi e aveva sempre un sorriso partecipe al clima che si era creato in quegli anni. Era arrivato a Napoli con le sue pubblicazioni, le sue competenze e con l'inconfondibile spirito fattivo. Il suo più grande contributo a quelle serate, e che ancora ricordiamo tra noi, fu una volta quando, a freddo, attorno alla tavola tonda e una montagna di pasta e vongole, chiese serafico se era vero che, come qualcuno gli diceva, aveva perso completamente il suo accento milanese. Lo disse, manco a dirlo, con il suo inconfondibile accento: qualcuno scoppiò a ridere, altri finsero di girarsi a prendere qualcosa per trattenersi e si andò avanti un paio di ore tra paradossi e risate che tirarono inevitabilmente dentro anche me e la mia calata bresciana, che vent'anni da napoletano hanno attenuato ben poco.

Non racconto questo per spirito di puro aneddoto, anche se sicuramente indurrà una risata da lassù lo stesso Massimo, ma per sottolineare una sua grande dote in un momento che era delicato e significativo per lui e per l'Accademia italiana. Quel suo posto di ricercatore universitario rendeva giustizia a una carriera non facile, ricca di pubblicazioni e competenze svariate, ma infranta contro concorsi in precedenza andati male, occasioni mancate e viaggi faticosi su e giù per l'Italia per contratti di insegnamento nelle varie discipline che sapeva insegnare con quel carisma che generazioni di studenti gli hanno sempre riconosciuto. A cinquant'anni compiuti Massimo poteva finalmente approdare in forma stabile nei ranghi dell'Università italiana e lo faceva con lo spirito ragazzino di chi vuole parlare, sorridere con gli altri e 
partecipare, direiquasi conumiltà, a quelloa cuiviene chiamato dalla sua Facoltà. Non è scontato, tanto meno oggi, in un'età di concorsi e carriere più stanziali. Massimo partiva ogni settimana da Milano e si fermava tutti i giorni necessari a insegnare, seguire laureandi che crescevano, partecipare alle incombenze organizzative, che cominciavano a pesare sempre più, e condividere anche momenti di svago con i colleghi che sempre più lo apprezzavano.

Nel momento in cui giunse Massimo, la Facoltà di studi arabo-islamici e del Mediterraneo era al suo apice, creata e costruita da Luigi Serra e poi guidata dal compianto Agostino Cilardo e in cui stavano, negli stessi anni, alcuni dei colleghi citati sopra, ma anche, in ambito arabo-islamico, Carmela Baffioni, Gioia Chiauzzi, Bartolomeo Pirone, Giuseppina Igonetti, Ersilia Francesca, Armando Salvatore e tanti altri.

La riforma Gelmini ha cancellato quell'esperienza unica ma non la consistenza degli studi napoletani di arabistica e islamistica, con tanti più giovani che sono arrivati e hanno sostituito i vecchi maestri. Massimo vi entrò con la sua storia già consistente, in un concorso che non fu affatto facile, non per le sue capacità indiscusse, ma per ritardi, folate di vento diverse che lo percorsero a più riprese, quando ancora le procedure erano nazionali.

Ci arrivò anche per l'incapacità del sistema universitario milanese di costruire qualcosa di solido e strutturato sulle competenze relative alle culture dell'Africa e dell'Asia. Oggi, sulla spinta dell'attualità, la realtà è un po' cambiata e forse Massimo troverebbe più facilmente una cattedra in uno degli atenei milanesi. È indubbio, tuttavia, che Milano ha perso un'occasione storica, che invece colse Venezia Ca' Foscari negli anni '6o del xx secolo, aprendo il corso di Lingue Orientali e guardando a un futuro orientale inevitabile. Da lombardo e grande ammiratore del dinamismo e delle capacità di Milano, che ne fanno una città di respiro europeo unica, ho sempre trovato inspiegabile come non si sia mai saputo cogliere il significato strategico di queste competenze, non solo dal punto di vista culturale, ma soprattutto economico. Massimo è stato vittima di questo limite, oltre che delle tipiche miopie accademiche che spesso privilegiavano l'autoriproduzione o guardavano a questi argomenti con supponenza o sufficienza. Il riconoscimento dell'Accademia Ambrosiana è stato uno dei pochi segni di interesse milanese e il suo impegno profuso anche in questa istituzione dimostra quanto l'avesse reso felice.

Massimo è stato un autore prolifico, anche proverbiale nella sua capacità di produzione in svariati campi di indagine. Poco amante della minuzia filologica raccolta in articoli scientifici, la sua penna facile ha sempre preferito il più agevole spazio della monografia, della traduzione commentata, della raccolta a sé di contributi di colleghi e dei numerosi allievi spesso attratti dal suo carisma. 
Tre sono state le grandi direttrici di suo interesse, oltre a svariati interventi in ogni direzione della storia del pensiero e della tradizione islamica - la filosofia, il Corano e la storia contemporanea - che alla fine si possono riassumere in un'unica grande passione per la vicenda culturale islamica nel suo complesso. La solida preparazione filosofica si può considerare la sua vocazione formativa e destinata a influenzare tutta la visione successiva. Sulla laurea in Filosofia ottenuta nell'anno accademico 1976-1977 alla Statale di Milano, con una tesi su Giordano Bruno, raccontava, aveva aggiunto un repentino e travolgente interesse per il Corano, letto in traduzione e poi portato al centro dei suoi interessi che lo avevano guidato a intraprendere lo studio dell'arabo che a Milano, in quegli anni, volevano dire il diploma in lingua araba presso la Sezione Lombarda (Milano) dell'Is.M.E.O, ottenuta nel 1983-1984. La formazione filosofica, a ben vedere, ne ha determinato attitudini e lettura di ogni aspetto della civiltà islamica e ha anche incanalato la natura della sua produzione, così attenta a una ricostruzione razionale e argomentata di un pensiero, di un'opera o di una vicenda storica che fosse.

Tra le sue pubblicazioni, inevitabilmente la storia della filosofia e la filosofia in genere hanno una posizione privilegiata. In particolare non si può, in questo ambito, non menzionare i suoi lavori su Averroè, che lo hanno accompagnato tutta la vita. ${ }^{1} \mathrm{Di}$ stretta e anche antica derivazione dai primi interessi filosofici va annoverata la passione per il pensiero di al-Ġazālì (m. 1111), che rappresenta la giusta sintesi tra competenze in storia del pensiero filosofico e un interesse più ampio islamistico. ${ }^{2}$ Penso che Massimo si rivedesse un po' di al-Ġazālī, nella sua fondamentale matrice filosofica eppure innovativa, perché portatrice nel suo percorso delle esigenze della tradizione religiosa. Il percorso di Massimo, i suoi interessi di studio non meno della sua vera partecipazione al tutto della civiltà islamica, vi si rispecchiava ampiamente.

Il rapporto tra ricerca e partecipazione razionale ed emotiva a una vicenda ritorna, io credo, anche nei suoi studi suòl pensiero di Ḥasan Ḥanafĩ che lo

1 Introduzione, traduzione e note ad Averroè. Il Trattato Decisivo. Milano, Bur Rizzoli, 1994; introduzione, traduzione e commento di Averroes. The Decisive Treatise. Piscataway NJ, Gorgias Press, 2017; introduzione, traduzione e note ad Averroè. L'Incoerenza dell'Incoerenza dei filosofi. Torino, Classici della filosofia U.T.E.T., 1997. Di carattere manualistico e più generale è La filosofia islamica. Brescia, La Scuola, 2016.

2 Da "Una via a Dio nel pensiero mistico di al-Ghazâlî". Rivista di Storia della Filosofia, 46/3 (1991), p. 463-479; "Al-Ghazâlî ed Anselmo: elementi di confronto", Rivista di Storia della Filosofia, 48/3 (1993), p. 611-623; ai più recenti "In Defence of Sunnism: al-Ghazali and the Seljuqs". In: The Seljuqs. Politics, Society and Culture. C. Lange e S. Mecit (ed.), Edinburgh, Edinburgh University Press, 2011, p. 228-239; Traduzione, introduzione e note ad al-Ghazali. Le luci della sapienza. Milano, Oscar Classici Mondadori, 2012; e Al-Ghazālì and the Divine. Series Islamic Philosophy. Abingdon-New York, Routledge, 2018. 
ha pure accompagnato negli anni. ${ }^{3}$ Ne parlavamo e ci scherzava su, giocando i ruoli abituali: io facevo lo scettico trattenendomi quel pesante sarcasmo che tra lombardi avrebbe comunque funzionato, ma lo facevo per rispetto di quello che sapeva argomentare come pochi, spesso risultando convincente.

L'interesse combinato per il pensiero islamico e per il politico trova, a mio parere, il suo prodotto per elezione nel suo libro dedicato a Islam e politica. $\grave{E}$ sempre stato uno dei miei preferiti ed è rimasto per quasi un paio di decenni tra le letture consigliate dei miei corsi di islamistica, raccogliendo grandi attenzioni dai lettori. Ho spesso inserito nelle letture consigliate molti suoi libri, sempre scelti dagli studenti, che quasi, sollecitati a dire quale libro avessero apprezzato maggiormente tra quelli letti a scelta, molto spesso indicavano qualcosa di Massimo. Islam e politica è più di un manuale: è un'efficace sintesi su un tema che specialmente negli ultimi anni ha sempre più richiamato l'attenzione di studiosi e ricercatori, argomentata in modo chiaro e anche con idee forti. ${ }^{4}$

Massimo ha sempre dichiarato che i suoi interessi islamistici e la sua vera e propria passione per l'Islam gli derivò dall'impressione che gli procurò, quando già era avviato agli studi filosofici, la lettura del Corano. Inevitabile, con questa premessa che il Corano, la storia della sua interpretazione e quindi dell'ermeneutica coranica occupassero uno spazio significativo nella sua bibliografia. Questa sua produzione ha avuto particolare eco anche al di fuori dei confini italiani dove Massimo è conosciuto in primo luogo come uno studioso di esegesi coranica contemporanea. ${ }^{5} \mathrm{Di}$ tutta questa produzione, che troneggia con pagine consumate nei miei scaffali di studi coranici, vorrei ricordare però due libri che mi capita non di rado di sfogliare, quasi a carpirne il fascino e

3 A partire da "Hasan Hanafi e la fenomenologia: per una nuova politica dell'Islam". Oriente Moderno, 84 (1994), p. 103-120.

4 Islam e politica. Bologna, Il Mulino, 1999, $2003^{2}, 2015^{3}$ (terza edizione rivista ed aggiornata). Trad. spagnola Islamy politica. Madrid, Biblioteca Nueva, 2003. Su questi temi Massimo è tornato anche in Oltre la democrazia. Temie problemi del pensiero politico islamico. Milano-Udine, Mimesis, 2014.

5 Vedi ad es. Il Corano e la sua interpretazione. Roma-Bari, Laterza, 2004; L'esegesi musulmana del Corano nel secolo Ventesimo. Brescia, Morcelliana, 2008 (traduzione ingl. ampliata e rivista The Qur'an: modern Muslim interpretations. London and New York, Routledge, 2011); Philosophical Perspectives on Modern Qur'anic Exegesis. Key Paradigms and Concepts. Sheffield UK - Boston, ст Equinox Publishing, 2016; introduzione, traduzione e note ad al-Ghazâlî. Le perle del Corano. Milano, Rizzoli, 200o. Su ermeneutica e Corano, vedi ad es. "Qur'an and Science: a Hermeneutical Approach". Journal of Qur'anic Studies, 7 (2005), 1, p. 48-64; "Qur'anic Hermeneutics and Political Hegemony: Reformation of Islamic Thought". The Muslim World, 99 (2009), 1, p. 124-133. 
l'attrattiva, per me, appassionato delle minuzie filologiche, alquanto misteriosa. Sono i suoi libri sulla sura della Caverna (Cor. Cap. 18) e sulla figura di Giuseppe. Li leggo e trovo sovvertito tutto il mondo di analisi dotte e minuziose e rimango avvinto da un pensiero e una chiave interpretativa solide, in fondo il suo inconfondibile marchio di fabbrica. ${ }^{6}$

Massimo ha avuto anche alte vite scientifiche e molteplici interessi culturali. La passione per la manualistica o per la saggistica per il pubblico non specialistico, che gli era particolarmente congeniale, è ben rappresentata dai suoi libri pubblicati per la casa editrice il Mulino. In questo genere di prodotti ritroviamo un altro suo settore di elezione, ovvero la storia contemporanea dei paesi arabi e dell'Egitto in particolare. ${ }^{7}$ L'origine di tale interesse risiedeva nel desiderio, credo, di ricostruire quell'ambiente storico così politicamente ricco che segnò la storia egiziana nel cuore del Xx secolo e che si saldò fin da subito con l'attenzione per le vicende dei Fratelli Musulmani che furono protagonisti di quella stagione. ${ }^{8}$

Ritroviamo in tutto questo molto della passione di ricerca ma anche personale di Massimo: la fascinazione per il Corano e per la vicenda storica e religiosa dei Fratelli Musulmani sono stati vissuti con una partecipazione spesso simpatetica, ad esempio fino agli sviluppi della breve stagione di ribalta dopo le primavere arabe. Ed era anche una sua passione politica, mai a incidere nel discorso scientifico ma che però alimentava anche lo sguardo indagatore, in una ricerca di senso che ha guidato molti studiosi del mondo arabo e islamico della generazione precedente, della sua e anche della mia. Linteresse politico, seppur coniugato con linee filosofiche, filologiche o storicistiche, si misura con esigenze diverse: dal riscatto di visioni pregiudiziali e riduttive, dal desiderio di una neutrale valutazione a una partecipazione simpatetica della vicenda culturale islamica nel suo complesso. Molti studiosi e ricercatori (come il Sottoscritto) si accontentano di una sola di queste motivazioni e spinte, Massimo al contrario era un po' tutto questo, perché infiniti erano i suoi interessi e stimoli intellettuali, ancor più corposi della sua bibliografia. ${ }^{9}$

6 La Sûrah della Caverna. Meditazione filosofica sull'Unicità di Dio. Pubblicazioni della Facoltà di Lettere e Filosofia dell'Università di Milano, n. Cxxv, Firenze, La Nuova Italia, 1986; Il Profeta Giuseppe. Monoteismo e storia nel Corano. Brescia, Morcelliana, 2007.

7 Vedi in particolare La teoria del socialismo in Egitto. Palermo, Centro Culturale al-Farabi, 1987; Storia del Medio Oriente contemporaneo. Bologna, Il Mulino, 2014; la curatele del volume "Arab Nationalism(s) in the Twentieth Century". Oriente Moderno, 1 (2017); Storia dell'Egitto dalla conquista araba ad oggi. Bologna, Il Mulino, 2017.

8 Vedi ad es. L'Alternativa islamica. Aperture e chiusure del radicalismo. Milano, Bruno Mondadori, 2012.

9 Su questo e quel che pensava sull'Islam Massimo è stato particolarmente esplicito in "La mistica islamica come problema critico". Introduzione a L. Gardet e G. Anawati. La mistica islamica. Milano, Jacabook, 2017. 
Gli ultimi dieci anni, con l'apparire dei suoi problemi di salute, la bibliografia di Massimo è letteralmente esplosa. Già autore prolifico, con una facilità di penna unica, è come se le difficoltà fisiche, ormai visibili a tutti, gli dessero ancora più forza. Va a suo merito questo sforzo di dire e scrivere tutto quello che si prefiggeva. Oltre che per il buon umore costante tra i suoi viaggi ad inseguire il riconoscimento accademico che tardava, Massimo è stato un esempio unico anche in questo. Non mancavano mai battute, da parte sua, sui suoi tremori e mai che questo fosse un motivo per commiserarsi e distogliere la mente dalla sua fame di conoscere e far conoscere, prima di rilassarsi insieme a tutti per una buona cena con una buona bottiglia di vino (ben poco islamico questo, caro Massimo).

Questa travolgente spinta a interessarsi di tutto e a scriverne lo ha senz'altro reso ingombrante, così come la scarsa attitudine per le minuzie filologiche e gli specialismi ristretti. Ma è stato molto più di un alto divulgatore. Non ho potuto certo leggere tutto quel che ha scritto, compito sovrumano (perdona l'ennesima battuta, Massimo) quasi quanto scriverlo, ma sono sempre rimasto spiazzato dalla sua capacità di entrare a fondo, in profili ampi e documentati, con il raziocinio filosofico e l'occhio simpatetico. Si poteva esserne spesso convinti oppure no, ma difficile era smontarlo in qualche dettaglio, e questo rimarrà sempre un mistero con tutto quello di cui si è occupato. Poco prima che mancasse ho ricevuto la sua monografia sulla biografia su Maometto ${ }^{10}$ e il primo pensiero è stato quello di vedere un po' che mai avesse scritto anche su questo argomento, un argomento che in genere riempie la vita di un singolo studioso. Anche questa volta ho chiuso il libro dovendo ammettere che sì, Massimo, anche su questo aveva qualcosa da dire, non banale, e ha saputo articolarlo e costruirlo come se non avesse fatto altro nella sua attività di ricerca.

Sono giunto alla fine di questo breve percorso, così come Massimo è giunto troppo presto alla fine del suo percorso di vita. Massimo ci accompagna e mi accompagnerà di sicuro in quelle passeggiate memorabili per ogni appassionato lettore e studioso che sono gli scaffali delle grandi biblioteche nordamericane e anche quelle, meno fornite, di tanti altri paesi. In quei luoghi insostituibili, anche se a volte eccentrici per scelte di classificazione, uno può trovare tutto o quasi tutto quanto si è scritto su ogni argomento. Quando

10 Maometto, l'Inviato di Dio. La vita e il messaggio di Muhammad, il profeta dell'Islam. Roma, Salerno Editrice, 2020. 
si attraversa le file di raccolte di studi su Islam, storia e filosofia islamiche a predominio anglofono uno dei pochissimi nomi italiani che si legge è sempre quello di Massimo Campanini. Lì rimarrà di sicuro a lungo, proprio come i buoni classici a sorreggere studenti e ricercatori, in Italia e nel mondo, a inorgoglire chi come me lo ha conosciuto e lo ha apprezzato come amico.

Ti chiedo scusa un'ultima volta ma non ce la faccio a non ricordare una delle ultime risate napoletane fatte insieme. Proprio in quel Palazzo Dumesnil dove sta il Rettorato e dove troneggia quella vista del mare di Napoli che fa dimenticare ogni tribolazione e, per te, ogni faticoso viaggio su e giù per l'Italia. Qualche anno fa, in occasione di un convegno proprio a Palazzo Dumesnil, mi annunciasti che avevi la possibilità di vincere un concorso da Professore Associato bandito dall'Università di Trento. Stavamo aspettando sedendo in prima fila l'inizio, scambiandoci battute e freddure a getto continuo da buoni lombardi - pur con le necessarie finte differenze tra un milanese cittadino e un valligiano di provincia bresciana come mi proclamavo sempre davanti a te per sollecitare il suo Vaffa bonario. Con quel garbo che nel nostro ambiente, in cui ognuno si sente spesso sempre in credito con le istituzioni e il mondo accademico, e raramente in debito, mi hai chiesto che ne pensavo di questa possibilità. Il garbo era dovuto al fatto che "l'Orientale" gli aveva finalmente dato un posto, non poteva ignorarlo e temeva di fargli e farci un torto. Questo fece Massimo Campanini con la sua bibliografia sterminata, con le prime edizioni inglesi dei suoi saggi e a cinquant'anni suonati. Noi tutti fummo subito contenti per lui, perché si avvicinava a casa e ne aveva bisogno e se questo era quello che voleva, andava bene. Lì per lì però non mi risparmiai l'ennesima freddura e sorridendo gli dissi "Massimo, per me non c'è problema, tanto si sa, sono i migliori i primi ad andarsene". Massimo rise rumorosamente, si toccò parti che è meglio non nominare e mi apostrofò con l'inevitabile Vaffa.

Oggi guardando verso il mare, dallo stesso posto, giusto un piano sopra, quella battuta prende un altro significato, ma non suona affatto irriverente. So che starà ridendo e, forse, tutto gli sembrerà più lieve dopo tanto viaggiare, tanto scrivere, tanto discutere e tanta passione riversata nelle cose che faceva, nel parlare di moglie e figlio, della sua Milano, dei tanti progetti futuri e di una unica a carismatica voglia di vivere che irradiava su tutti coloro che gli stavano intorno. Alla fine, se ci penso, sono orgoglioso di quella battuta ritrita perché nel suo caso annunciava proprio una grande verità: purtroppo alla fine sono proprio i migliori come lui i primi ad andarsene. 